\title{
The Spatial Spectrum Of Tangential Skin Displacement Can Encode Tactual Texture
}

\author{
Michael Wiertlewski, José Lozada, and Vincent Hayward Fellow, IEEE
}

\begin{abstract}
The tactual scanning of five naturalistic textures was recorded with an apparatus capable of measuring the tangential interaction force with a high degree of temporal and spatial resolution. The resulting signal showed that the transformation from the geometry of a surface to the force of traction, and hence to the skin deformation experienced by a finger is a highly nonlinear process. Participants were asked to identify simulated textures reproduced by stimulating their fingers with rapid, imposed lateral skin displacements as a function of net position. They performed the identification task with a high degree of success, yet not perfectly. The fact that the experimental conditions eliminated many aspects of the interaction, including low-frequency finger deformation, distributed information, as well as normal skin movements, shows that the nervous system is able to rely on only two cues: amplitude and spectral information. The examination of the "spatial spectrograms" of the imposed lateral skin displacement revealed that texture could be represented spatially despite being sensed through time and that these spectrograms were distinctively organized into what could be called "spatial formants". This finding led us to speculate that the mechanical properties of the finger enables spatial information to be used for perceptual purposes in humans without any distributed sensing, a principle that could be applied to robots.
\end{abstract}

\section{INTRODUCTION}

$\mathbf{T}$ EXTURE - the organized deviation from smoothness of the surface of objects - typically is first apprehended visually but once contact is made with the hand, touch must take charge. Katz, in 1925, noted that there are two ways in which organisms can become tactually aware of the texture of objects [1]. One way is to determine directly the relevant spatial features of the geometry of a touched surface. To illustrate how this could be done, consider a deeply grooved grating, such as a knurled knob. Under reasonable normal static loading, the skin interacts with such a surface through a collection of minute contact surfaces. Assuming that the sensory apparatus is able to detect these individual contact surfaces, then presumably a coarse notion of the surface geometry can be acquired. If such surface has any degree of fineness, however, the individual contacts become so numerous that such strategy becomes highly implausible. Most psychologists and neurophysiologists agree with Katz that the experience of surface texture must result from mechanical signals brought about by finger sliding that change through time, in addition to mechanical signals that vary through space.

M. Wiertlewski and J. Lozada are with CEA LIST, Sensory and Ambient Interfaces Laboratory, Fontenay-Aux-Roses, France. E-mail: michael.wiertlewski@cea.fr, jose.lozada@cea.fr. V. Hayward is with UPMC Univ Paris 06, UMR 7222, Institut des Systèmes Intelligents et de Robotique, Paris, France. E-mail: vincent.hayward@isir.fr.

Vol. 27, No. 3, pp. 461-472. doi 10.1109/TRO.2011.2132830
To the haptics engineer interested in devices and transducers able reproduce tactile and haptic sensations, these observations are very significant since the overriding objective is to extract from the complexity of the ambient physics those aspects that are the most significant to the perceiver and to discard the others in the name of technical feasibility.

Of course, tactual texture is an ill-defined notion. In a single sentence, it is hard to discuss the sensations caused by rough burlap, those resulting from finely machined bronze, or those derived from the velvety skin of an apricot. To make things worse, from a physical view point, and even restricting attention to hard materials, texture and roughness can be characterized in many different ways that also depend on the method used to measure it [2], [3]. With soft materials the situation is even more inextricable.

The many studies in the psychophysics of texture and roughness perception unfortunately contribute little insight to the haptics engineer because these studies rarely speak of the same quantities, although there is a general agreement that roughness has perceptual significance [4], [5], [6], [7], [8], [9], [10], [11], [12], [13], [14], even if it is nearly impossible to define it unambiguously from the physical characteristics of the touched object [15].

If roughness, and more generally, if tactual texture is hard to discuss directly from the physics of an object, then perhaps a more productive approach from the view point of interface design would be to focus on the characteristics of the mechanical interaction of the skin with an object, although the prospects for identifying simple signals are rather bleak at first sight. The finger is a soft, highly deformable object which, besides its complex detailed geometry, exhibits several types of nonlinearities that are manifest at different length scales of interaction with surfaces [16], [17], [18], [19], [20]. Even under the extremely simplified assumption of linear viscoelasticity and perfectly clean contacts free of foreign bodies and liquids, the contact of deformable bodies with rough surfaces gives rise to theories of considerable complexity that are unlikely to yield simple interaction models [21]. These observations justify the measurement-reproduction approach adopted in this study.

\section{ROUghnesS AND TEXTURE IN MANUfACTURING AND ViRTUAL REALity}

Numerous industrial processes, from mirrors to roads, depend on the measurement of roughness. It is achieved using profilometers based on slow mechanical scanning with a sharp stylus (the tip radius can be as small as a few nanometers) or 
by optical methods (confocal microscopy, laser triangulation, interferometry). Reporting roughness is mostly a function of the intended application. In part machining, for instance, roughness is traditionally characterized in terms of the relative heights of a set of asperities specifying their standardized moments: $0^{\text {th }}, 1^{\text {st }}, 2^{\text {nd }}, 3^{\text {rd }}$. Interestingly, the latter measures report zero roughness for any regular grating and therefore cannot be applied to perceptual studies. Other measures report the statistics of the peak-to-valley distances of sets of asperities which makes them more relevant. Some measures consider autocorrelation, some account for spatial wavelength or for extrema density. Some others take into consideration the magnitude of the slopes of asperities, and yet others their curvature. The later measure is probably one of the most relevant to tactual roughness of these different approaches.

The measurement process is typically slow (minutes, hours) and provides details that are not necessarily relevant to tactual sensing. On the other hand, it is an everyday experience that the roughness of a surface can be felt, or that two textured surfaces can be discriminated, or even that a wood grain can be identified in a fraction of a second by the scanning finger.

These observations have let researchers in virtual reality to adopt the more expeditious method used by humans to sense texture, rather than to rely on industrial-type methods. Examples of this approach can be found in [22] where the scanning interaction force is measured, in [23] where the scanning acceleration of a stylus is measured, or in [24] where the scanning velocity is measured. The reader is referred to a recent survey where 50 articles on the subject are commented [25].

For texture reproduction, the most widely adopted approach is the force-feedback device with a position-dependent textured virtual wall, also extensively surveyed in [25]. A more recently introduced technique is to modulate the friction force between the finger and a mechanically grounded active surface. The friction force modulation can be achieved, for instance, by electrostatic fields [26], ultrasonic amplitude modulation [27], surface acoustic waves [28] or with the squeezed film effect [29], [30].

\section{Design Motivation for A Texture Transducer}

The above considerations led us to engineer a new device capable of accurate measurement and reproduction of a surface-finger interaction, having a bandwidth and a dynamic range able to do justice to the biomechanics and sensory performance of the human finger. This device is already briefly described in reference [31] where is was shown that it was able to provide perceptually equivalent sensations of roughness between a virtual and a real surface. The surface used in these preliminary experiments was a "simple" triangular grating of spatial period $1.0 \mathrm{~mm}$ with groove depth $0.1 \mathrm{~mm}$. Although the surface was periodic, the force of interaction during sliding turned out to be a complex, broadband signal having a complicated harmonic signature which can be appreciated by consulting Fig. 1 and caption. The transformation from geometry to signal is highly nonlinear, a fact that is hardly surprising considering that friction is the primary phenomenon involved [32].

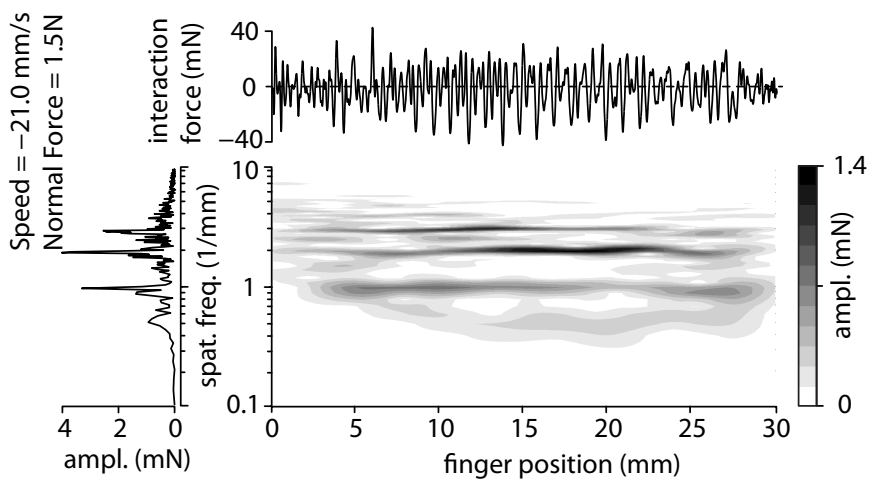

Figure 1. Spatial spectrogram produced by sliding a finger on a perfectly periodic triangular grating. The methods used to construct such plots are described in detail later in this article. For now, it can be appreciated that the transformation from a triangular profile to a force signal is far from straightforward. A triangular wave has only odd harmonics. While the fundamental "formant", or spectral peak, at $1 \mathrm{~mm}^{-1}$ is present, it is actually weaker than the first even-harmonic spectral peak. Notice also the present of energy in the sub-harmonic frequencies. These are the hallmarks of a nonlinear transformation.

In the present article, we describe this device in greater detail and we employ it in a experiment where it is used to reproduce various textures. We show that the textural recordingreproduction obtained with this device is of sufficient quality to enable several participants to correctly match a virtual surface with a real surface included in a set of five. The mechanical consequences of net friction were eliminated by the transduction process and so was distributed skin deformation within the finger contact area. As a result, the apparatus reproduced accurately the oscillatory components of the skin tangential displacement at the exclusion of other mechanical consequences of sliding a finger over a rough surface.

A particular feature of our device is that the same mechanical structure was used in the sensor and actuator modes. It is based on the piezoelectric effect which, as is well known, is reversible. In sensor mode it operates like a high-quality, stiff force sensor. In actuator mode it provides accurate isometric stimulation to the skin. The questions regarding the reciprocal signal causalities are discussed in [31].

\section{A. Performance Considerations}

The device should be orders of magnitude stiffer than the fingertip to provide unambiguous measurement and stimulation, noting that the converse possibility is considerably harder to achieve due to the difficulties met in reducing the effects of inertia to sub-threshold levels [33].

Other design considerations include the level at which interaction forces should be resolved. In absence of knowledge on the smallest dynamic forces able to stimulate the skin, an estimate can be obtained by considering that the elasticity of the fingertip is roughly of the order of $10^{3} \mathrm{~N} \cdot \mathrm{m}^{-1}$ and that a detectable skin displacement is of the order of $10^{-7} \mathrm{~m}$. One could infer that the sensor should resolve $10^{-4} \mathrm{~N}$, which is far beyond the reach of commercial strain-gauge force sensors. In terms of actuator displacement, similar considerations indicate that $10^{-4} \mathrm{~m}$ would be needed to create the $10^{-1} \mathrm{~N}$ peak-topeak force oscillations that can be encountered when stroking 
texture as can be seen from Fig. 1. This requirement has been, in hindsight, the hardest to meet and, due to saturation, has somewhat limited the scope of our investigations. Finally, it is commonly accepted that a $500 \mathrm{~Hz}$ bandwidth is needed to represent tactile interactions. Interestingly, this figure was actually proposed by Katz almost a century ago [1].

\section{B. Description}

The main components, shown in Figure $2 \mathrm{a}$, comprise a multilayer, $40 \mathrm{~mm}$ piezoelectric disk-bender (CMBR07, Noliac Group A/s, Kvistgaard, Denmark) connected to a $20 \mathrm{~mm}$ wide tray that can hold a sample. The bender is clamped vertically by two epoxy ridges of semi-circular section that apply uniform pressure on the bender. A treaded rod connected to the hollow center of the bender transmits motion to the tray which is linearly guided by a flexure made of two leaf springs. Connection to the bender is realized by two Delrin ${ }^{\complement}$ washers that can tolerate $\pm 0.5^{\circ}$ of misalignment. The texture samples are bonded to the tray using double-sided adhesive tape.

During sensor operation, the interaction forces induce flexural deformations of the blade along $x$ and in the piezoelectric element, as indicated in Fig. 2b. Through the piezoelectric effect, the deformation of the ceramic causes charges to appear on the electrodes that are picked up by an instrumentation amplifier. Conversely, when applying a voltage to the electrodes of the bender, the piezoelectric effect causes the transducer to operate as an actuator. In this case, displacements of the tray impose deformations in the fingertip resting on it.

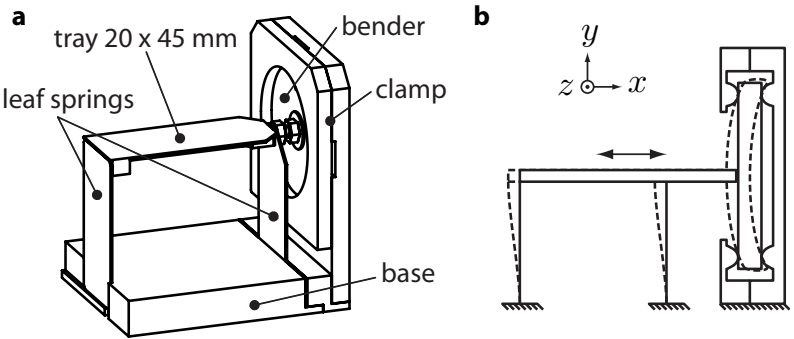

Figure 2. a. Transducer schematic. b. Cross section of the system at rest (solid lines) and during deformation (dashed lines).

\section{Transducer Modeling}

Since the mechanical constitution of the transducer is common to the sensor and to the stimulator, their models include the same lumped parameters. They differ only by the electronics. In sensor mode, a high gain, low noise instrumentation amplifier collects charges and convert it into readable voltage, whereas in actuator mode, a high voltage amplifier, with a low output impedance is used to drives voltage on the electrodes of the piezoelectric bender and therefore the tray's displacement.

1) Mechanical Flexure: The flexure acts like a mass-springdamper system with stiffness $k_{r}$ according to Fig. 3a. Damping due to internal and external friction is by and large dominated by losses in the bender. It arises mostly from hysteresis in the piezoelectric material. As further discussed later, it is reasonable to represent it by viscous damping. The inertial term corresponds to the equivalent moving masses of the tray and of the bender. The actuator force is shown as an external force, $f_{p}$, acting in the opposite direction of $x$. Another external force, $f_{d}$, models the finger interaction through its contact with the sample.
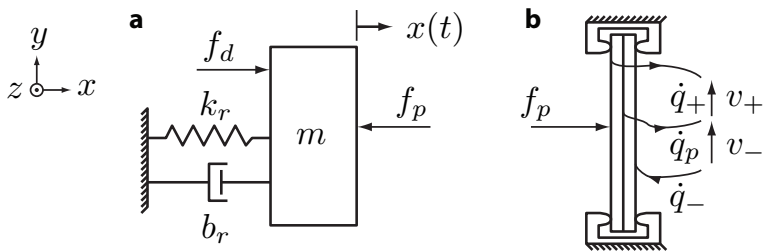

Figure 3. a. The plate, the sample and the bender are modeled by a mass $m$. It is suspended by a spring of stiffness $k_{r}$ connected to a damper $b_{r}$. Forces $f_{p}$ and $f_{d}$ represent the piezoelectric actuator and finger interaction forces, respectively. b. The piezoelectric effect causes charges to appear on the electrodes as a result of displacement.

Applying Newton's second law and converting to the Laplace domain gives

$$
\left(m s^{2}+b_{r} s+k_{r}\right) X(s)=-F_{p}(s)+F_{d}(s),
$$

where $X(s), F_{p}(s)$ and $F_{d}(s)$ represent the Laplace transform of the variables $x(t), f_{p}(t)$ and $f_{d}(t)$.

2) Static Constitutive relationships: The Y-poled bimorph piezoelectric element has two external electrodes plus one central electrode located in the neutral fiber. Bending deformation results in the compression of one layer and traction of the other. Layers are polarized which creates charges $q_{+}, q_{p}$ and $q_{-}$through the piezoelectric effect. Operating as an actuator, imposed voltages $v_{+}$and $v_{-}$push the charges on the armatures to induce axial deformation as a result of the radial strain. The linear, static constitutive relationships can be expressed in matrix form as follows [34],

$$
\left(\begin{array}{c}
x \\
q_{+} \\
q_{-}
\end{array}\right)=\left(\begin{array}{ccc}
1 / k_{p} & \beta & \beta \\
\beta & C_{\mathrm{p}} & 0 \\
\beta & 0 & C_{\mathrm{p}}
\end{array}\right)\left(\begin{array}{c}
f_{p} \\
v_{+} \\
v_{-}
\end{array}\right),
$$

where $\beta=\delta_{\max } / 2 v_{\max }$ is the ratio of the largest unloaded deflection to the total maximum operating voltage applied to one layer, $k_{p}$ is the flexural stiffness in open circuit and $C_{\mathrm{p}}$ is the capacitance of one piezoelectric layer when no stress is applied. In this model, the dynamic parameters like mass and damping are not taken into account.

3) Transfer function in sensor mode: Only one layer is used. The voltage generated by an external force can be written from (2) by summing the piezoelectric induced voltage, $v_{\mathrm{p}}$, with the voltage due to the circulation of charges,

$$
v_{+}=\frac{q_{0}+\int \dot{q}_{+} \mathrm{d} t}{C_{\mathrm{p}}}-\frac{\beta}{C_{\mathrm{p}}} f_{p}=\frac{q_{+}}{C_{\mathrm{p}}}+v_{\mathrm{p}} .
$$

The transducer acts electrically like a voltage generator in series with a capacitor $C_{\mathrm{p}}$. The generated voltage, $v_{+}$, is amplified by a high input impedance $\left(10^{12} \Omega\right)$ instrumentation amplifier (LT1789, Linear Technology Corp., Milpitas, CA, USA), see Fig. 4. Load resistances, $R_{s}$, combined with the 
capacitor form a first-order high-pass filter which can be expressed in the Laplace domain by

$$
V_{+}=-\frac{2 \beta R_{s} s}{1+2 R_{s} C_{\mathrm{p}} s} F_{p}=\frac{2 R_{s} C_{\mathrm{p}} s}{1+2 R_{s} C_{\mathrm{p}} s} V_{\mathrm{p}}
$$

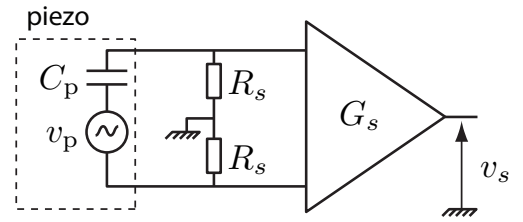

Figure 4. Schematic of the sensor circuit. The electrode of the upper layer is connected to an instrumentation amplifier. Resistors create a high-pass filter.

Neglecting the contribution of $v_{\mathrm{p}}$ to the mechanical behavior, the mechanical constitutive equation is $x(t)=1 / k_{p} f_{p}(t)$. Combining it with (3), $V_{s}(s)=G_{s} V_{+}(s)$ gives the output voltage, $V_{s}(s)$, as a function of the displacement $X(s)$ :

$$
V_{s}(s)=-G_{s} \beta k_{p} \frac{2 R_{s} s}{1+2 R_{s} C_{\mathrm{p}} s} X(s)
$$

Using (1), the transfer function of the sensor, $H_{s}(s)$, becomes

$$
H_{s}(s)=\frac{V_{s}(s)}{F_{d}(s)}=\frac{-2 G_{s} R_{s} k_{p} \beta s}{\left(1+2 R_{s} C_{\mathrm{p}} s\right)\left(m s^{2}+b_{r} s+k_{r}+k_{p}\right)}
$$

4) Transfer function in actuator mode: The bender is connected to a power source (PA86U, Cirrus Logic Inc., Austin, TX, USA) which drives the central electrode as in Fig. 5. The amplifier is connected voltage-mode with a gain $G_{a}=20$. A resistor, $R_{a}$, in series with the output tunes the frequency response since a low pass filter is formed with the capacitance of the piezoelectric element.

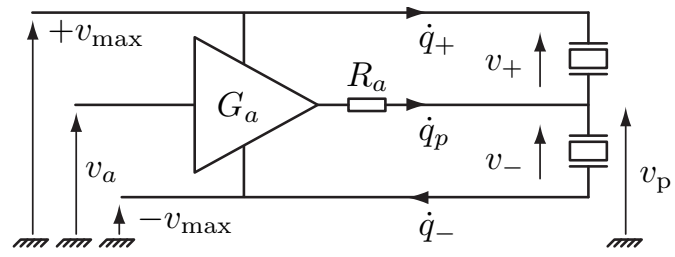

Figure 5. Circuit in actuator mode. Upper and lower electrodes are connected to fixed voltages $\pm v_{\max }$. The power amplifier drives the central electrode voltage, $v_{\mathrm{p}}$.

By application of Kirchhoff's law at the output node,

$$
\dot{q}_{+}+\dot{q}_{p}=\dot{q}_{-},
$$

with $\dot{q}_{+}=C_{\mathrm{p}} \dot{v}_{+}, \dot{q}_{p}=\left(1 / R_{a}\right)\left(G_{a} v_{a}-v_{p}\right)$ and $\dot{q}_{-}=C_{\mathrm{p}} \dot{v}_{-}$. Using these values in (4) and substituting $v_{+}=v_{\max }-v_{p}$ and $v_{-}=v_{p}+v_{\max }$ yields in the Laplace domain,

$$
\frac{1}{R_{a}}\left(G_{a} V_{a}-V_{p}\right)=C_{\mathrm{p}}\left(V_{p}+V_{\max }\right) s-C_{\mathrm{p}}\left(V_{\max }-V_{p}\right) s,
$$

finally giving,

$$
V_{p}=\frac{G_{a}}{1+2 R_{a} C_{\mathrm{p}} s} V_{a}
$$

The power stage acts as an amplifier of gain $G_{a}$ with a firstorder low-pass filter of cutoff frequency $\nu_{\text {cut }}=1 /\left(4 \pi R_{a} C_{\mathrm{p}}\right)$. The first line of (2) combined with (1) gives

$$
\left(m s^{2}+b_{r} s+k_{r}+k_{p}\right) X(s)=2 \beta k_{p} V_{p}(s)+F_{d}(s) .
$$

The two last lines of (2) can be simplified since the voltage driver supplies and draws charges as necessary such that (2) becomes

$$
q_{+}=C_{f} V_{+} \quad \text { and } \quad q_{-}=C_{f} V_{-} .
$$

The transfer function of the unloaded stimulator is found by combining (5) with (6),

$$
H_{a}(s)=\frac{X(s)}{V_{a}(s)}=\frac{G_{a} k_{p} \beta}{\left(1+2 R_{a} C_{\mathrm{p}} s\right)\left(m s^{2}+b_{r} s+k_{r}+k_{p}\right)} .
$$

\section{Identification}

The model parameters could be initially estimated from the data provided by manufacturers, as well as from the design of the leafs and of the tray. Parameter identification was then performed to obtain a better model and to take into account nonlinearities and parameter deviations from their manufacturing specification. Because the mechanical parameters are common to the sensor and the actuator, it is more convenient to identify the system first in actuator mode.

1) Actuator mode: The frequency response was determined using a frequency sweep from $10 \mathrm{~Hz}$ to $1000 \mathrm{~Hz}$, applying 4 Vpp voltage signal, $v_{a}$, (using a digital-to-analog converter PCI-6229, National Instruments Corp., Austin, TX, USA). Output displacement was measured using a laser telemeter (LT2100 with LC2210, Keyence Corp., Osaka, Japan). At each frequency, amplitude and phase were measured after a $200 \mathrm{~ms}$ pause to let the transients subside. The response was determined under the following conditions: unloaded, with a finger resting on the tray (normal force $\approx 0.5 \mathrm{~N}$ ) and with a finger pushing down the plate (normal force $\approx 1 \mathrm{~N}$ ). The result is shown in Fig. 6. The system exhibits the intended natural resonance at $500 \mathrm{~Hz}$ followed by a small un-modeled resonance at $800 \mathrm{~Hz}$.
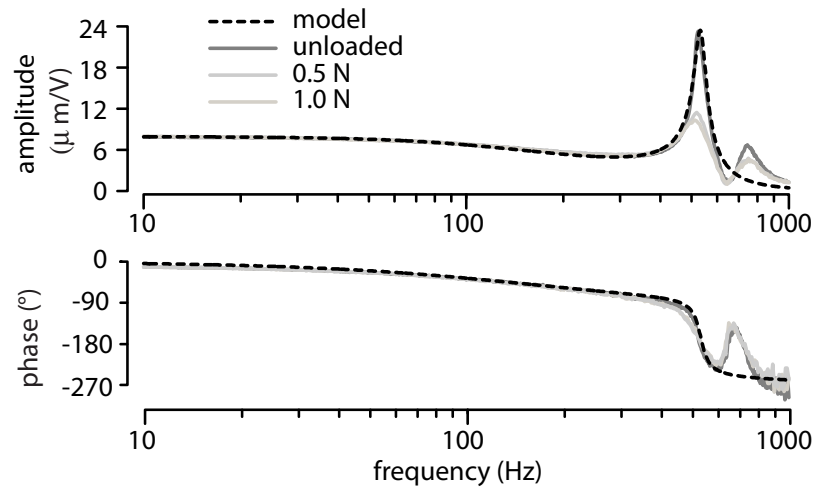

Figure 6. Frequency response of the actuator. Measurement are in grey dot and the model in plain black.

Since the actuator is two order of magnitude stiffer than the finger, finger loading has a negligible impact on response in a $10-400 \mathrm{~Hz}$ band. At resonance, however, damping due the 
fingertip causes a $3 \mathrm{~dB}$ attenuation of the resonant peak. In the experiments, caution was taken to roll-off the signal with $3 \mathrm{~dB}$ attenuation at $500 \mathrm{~Hz}$, flattening the response. The effects of the finger damping as well as of the second resonance can therefore be neglected. Least-square fitting $\left(R^{2}=0.85\right)$ provided the parameters shown in Table I. As can be seen from the figure, the model and the uncorrected system responses are graphically indistinguishable up to $500 \mathrm{~Hz}$.

Table I

ELECTRO-MECHANICAL PARAMETERS.

\begin{tabular}{l|l} 
Mechanical & Electrical \\
\hline$\delta=82.35 \mu \mathrm{m}$ & $C_{\mathrm{p}}=818 \mathrm{nF}$ \\
$k_{p}=76.47 \times 10^{3} \mathrm{~N} \cdot \mathrm{m}^{-1}$ & $v_{\max }=100 \mathrm{~V}$ \\
$m=6.8 \mathrm{~g}$ & $G_{s}=100$ \\
$b_{r}=4.49 \mathrm{~N} \cdot \mathrm{mm}^{-1} \cdot \mathrm{s}^{-1}$ & $R_{s}=12 \mathrm{k} \Omega$ \\
$k_{r}=4.05 \times 10^{-3} \mathrm{~N} \cdot \mathrm{m}^{-1}$ & $G_{a}=20$ \\
& $R_{a}=680 \Omega$
\end{tabular}

It is known that piezoelectric ceramic transducers have significant hysteresis which affects the quasi-static and the dynamic responses. Fig. 7 plots the response of the transducer to $0.1 \mathrm{~Hz}$ sinusoidal $10 \mathrm{~V}$ peak-to-peak amplitude signal showing a $16 \%$ hysteresis. The hysteresis introduced by piezoelectric ceramics is of non-saturating type and hence introduces small amplitude distortion of no consequence in our experiments, since the minor loops are very small. It does introduce constant phase lag of $8^{\circ}$ which, at a given frequency, can be represented as linear damping [35]. It is the actuator hysteresis that accounts for the nicely damped resonance of the system at $500 \mathrm{~Hz}$ but is neglected in the low frequences. In summary, the actuator is capable of a maximum peak-topeak displacement of $200 \mu \mathrm{m}$, with a quasi-static gain of 20 $\mu \mathrm{m} / \mathrm{V}$ in the range from DC to $500 \mathrm{~Hz}$.

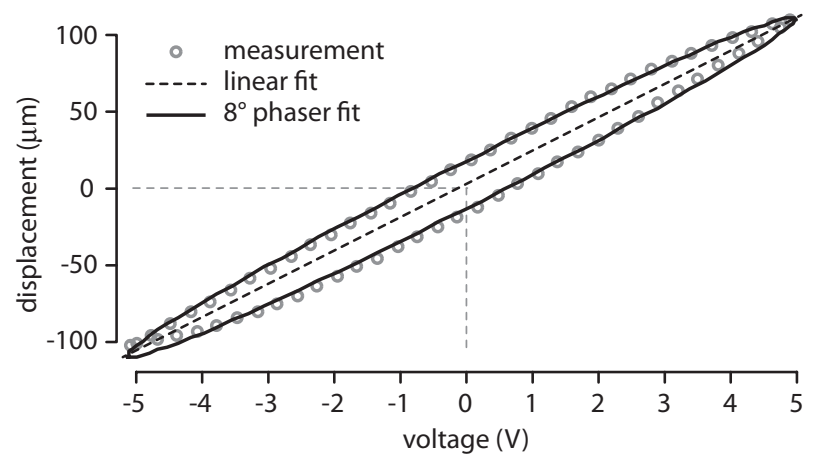

Figure 7. Quasi-static measurement of the actuator response (gray circles). Data show a non saturating hysteresis that can be approximate by a $8^{\circ}$ ideal phaser (black line).

2) Sensor mode: A known external force, calibrated using a conventional force sensor (Nano 17, ATI Industrial Automation, Apex, NC, USA), was applied to the sensor. This force was used as an input for the model described earlier. Fig. 8 the fit of the model with the measurement $\left(R^{2}=0.91\right)$. The simulated output is 10 times more noisy than the actual measurement from the sensor because of the noisy input measurements from the strain-gauge force sensor.

The model predicts a sensitivity of $26 \mathrm{~V} \cdot \mathrm{N}^{-1}$ for a gain of $G_{s}=100$ in the bandwidth $10-500 \mathrm{~Hz}$ with the resistor

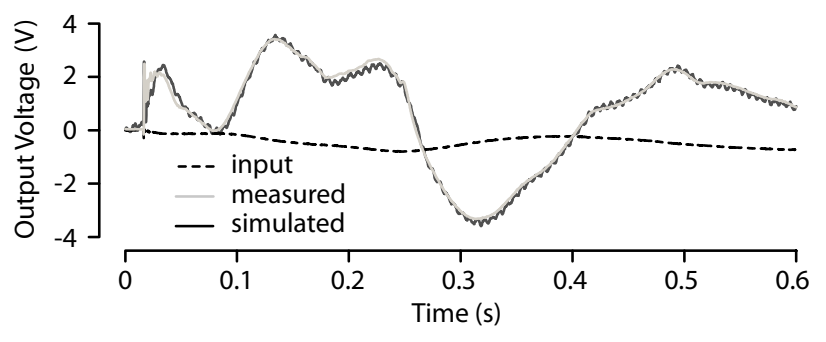

Figure 8. Fit of the sensor model with actual measurements.

$R_{s}$ set to $12 \mathrm{k} \Omega$. Like in the actuator mode, the sensor has a natural resonance at $500 \mathrm{~Hz}$. The signal is acquired with the data acquisition board already mentioned. With 16 bits of resolution, the force signal can be measured with $10^{-5} \mathrm{~N}$ resolution. The experimentally measured noise floor is as low as $25 \mu \mathrm{N} / \sqrt{\mathrm{Hz}}$.

\section{EXPERIMENT 1 : TEXTURE IDENTIFICATION}

As described in [31], the transducer was used in a causality inversion process: recording force and stimulating with displacement, but instead of asking participants to simply compare the roughness of a virtual surface with that of a real one, we asked them to identify different textures, thereby showing that they could discriminate textured surfaces in the complete absence of stimulation distributed in space. The principle of the experiment was to first record interaction with five different texture samples. A group of participants were then asked to identify three of these virtual samples among the five real samples, and another group of participants to match three of the real samples with the five real samples, leaving much possibility for confusion. We expected participants to be able to identify the real or the virtual textures with an equivalent level of performance. We also expected that a learning effect would be made apparent from the order of testing.

\section{A. Methods and Materials}

1) Design: We used a 5-alternative forced-choice matching procedure during which the participants were asked to identify a comparison stimulus with the five five standard textures. With the first group of five participants, in a first session, the comparison stimulus was a real surface picked randomly in a set of three and the standard stimuli were the five real textures. They were then tested in a second session with a simulated surface picked randomly among the same set of three as the comparison stimulus and the standard stimuli were also the five real textures. A different group of five participants was also tested, but with the simulated stimuli first, and then with real comparisons. The two missing textures in the comparison set served as 'catch samples' to test the participants' ability to detect non matching textures. They also acted as distractors since the participants were looking for them.

2) Sensing apparatus: The transducer was used to measure the interaction force of the author's finger sliding on a textured surface as in Fig. 9. The samples were placed on the tray of the 
transducer. The aforementioned strain-gauge based force sensor measured the low frequency components of the interaction force. The finger position was located by a precision a LVDT sensor (SX 12N060, Sensorex SA, Saint-Julien-en-Genevois, France) attached to the fingernail.
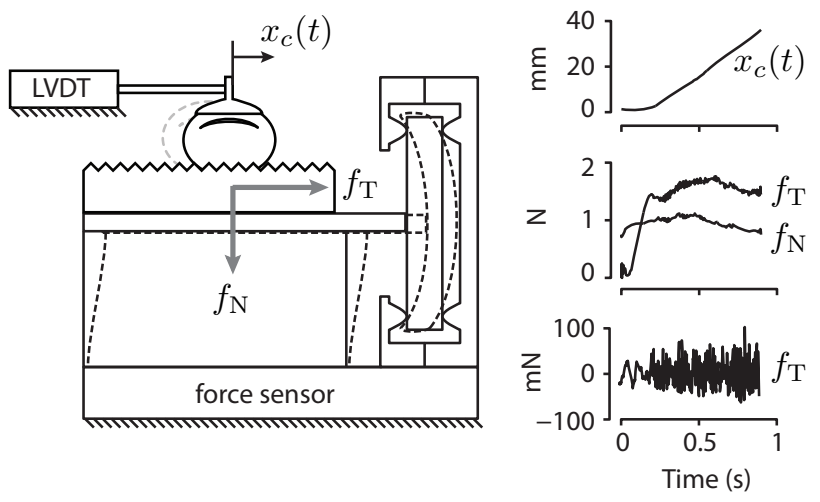

Figure 9. Sensor operation. A textured sample is bonded to the central plate. The finger position is measured by a LVDT sensor and the total interaction force monitored by a six-axis force sensor.

The position of the finger, the net force, and the tangential force sensed by the transducer were recorded at a $10 \mathrm{kHz}$, i.e. with sampling period $T=10^{-4} \mathrm{~s}$. The transducer signal was processed by computing its Fourier transform, truncating the spectrum to $15-500 \mathrm{~Hz}$ and then reconstructing the signal. This method ensured that the signal was restricted in the band where the transducer had a flat frequency response, excluding any artifact and phase distortion.

3) Stimulating apparatus: The friction force is correlated with the position of the finger, hence the signal is better expressed in the spatial domain and must be converted into this domain. Since the speed of a finger is of the order of $50 \mathrm{~mm} \cdot \mathrm{s}^{-1}$ the displacement resolution had to be better than $5.0 \mu \mathrm{m}$. An interpolation procedure was employed to reconstruct the signal in the spatial domain with the required resolution from the time-sampled force, $f_{\mathrm{T}}(j T)$, and from the time-sampled finger position $x_{c}(j T), j \in \mathbb{N}$. The discrete functions $x_{c}(j T)$ and $f_{\mathrm{T}}(j T)$ were first fitted with piecewise cubic Hermite functions, $\mathcal{S}_{x}(t) \simeq x_{c}(t)$ and $\mathcal{S}_{f}(t) \simeq f_{\mathrm{T}}(t)$, and then re-discretized. Given a space sampling period, $\epsilon$, position was resampled into samples $x_{i}=i \epsilon, i \in \mathbb{N}$. The force could then be represented in the discrete space domain following

$$
f_{\mathrm{T}}\left(x_{i}\right)=\mathcal{S}_{f}\left(\mathcal{S}_{x}^{-1}\left(x_{i}\right)\right) .
$$

The spatial sampling period was chosen to match the smallest step achievable with the apparatus, $\epsilon=1.0 \mu \mathrm{m}$.

The transducer was guided by a precision linear bearing, E, located by an encoder, F, (Model R119 Gurley Precision Inc, Troy, NY, USA) that could resolve position with $1.0 \mu \mathrm{m}$ precision. The fingertip rested on the tray, A, and the transducer tracked the position of the proximal phalanx, D, resting in a cradle, G, see Fig. 10a, relieving the fingertip from lateral loads. As the slider moved with the finger, the transducer stimulated the fingertip as shown in Fig. 10b. Each $0.5 \mathrm{~ms}$ the position of the slider was read, the software interpolated the drive signal from a pre-recorded texture profile.
A gain $G_{\psi}$ was adjusted to calibrate the stimulation for perceptually equivalent roughness with the real sample as described in [31]. To ensure that the drive signal matched the mechanical bandwidth of the transducer, an active analog $3^{\text {th }}$ order Butterworth filter with a cut-off frequency $600 \mathrm{~Hz}$ (Salen-Key configuration with amplifier OP2177, Analog Devices Inc., Norwood, MA, USA). The filter served as a signal reconstruction filter and also compensated for the mechanical resonance.

a

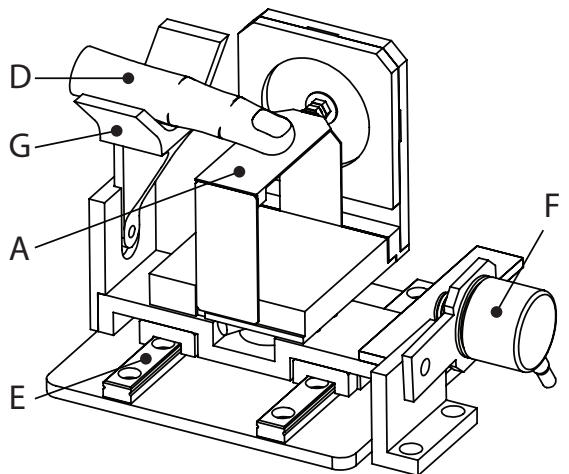

b

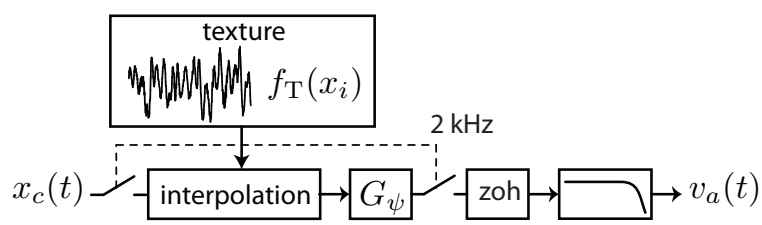

Figure 10. Stimulator operation. a. The transducer is mounted on linear bearing. The slider is located by an encoder. b. The control voltage is updated of piezoelectrical actuator as a function of the position and the texture profile used. The open loop command used.

Assuming that the Fourier transform of the texture signal exists, the accuracy of its restitution can be analyzed in terms of the maximum achievable spatial frequency for particular speeds, $\dot{x}_{c}(t)$, of the finger. The signal generated digitally was filtered at $\nu_{\text {cut }}=500 \mathrm{~Hz}$. It had for effect to limit the largest reproducible spatial frequency to, $\mathcal{F}_{\max }=\nu_{\text {cut }} / \dot{x}_{c}$ [36]. Assuming a finger velocity of $50 \mathrm{~mm} \cdot \mathrm{s}^{-1}$, the finest represented grating had a $0.1 \mathrm{~mm}$ period. The spatial resolution of the device was $\epsilon=10^{-3} \mathrm{~mm}$, the largest spatial frequency was $\mathcal{F}_{\text {max }}=10 \mathrm{~mm}^{-1}$ so the textures were represented by at least 100 samples per period. At higher speeds, the temporal sampling became the limiting factor. In terms of the Nyquist sampling frequency, the fastest variations were reconstructed with at least $\dot{x}_{c} /\left(\mathcal{F}_{\max } T\right)=12$ temporal samples.

This analysis shows that the device could reproduce spatial grating as low as $0.1 \mathrm{~mm}$, with high fidelity and a low sampling noise, both in spatial and temporal domain.

4) Test bench: In order to ensure that the matching of virtual and real textures were performed under conditions that were as similar as possible, a test bench was constructed where the simulated and the real surfaces had to be touched under the same constraints, see Fig. 11a. In the two conditions, the user's finger was resting on a cradle that was attached to the stimulator. Since all moving parts were rigidly attached, participants felt the same inertia and the same mechanical 
imperfections in the two conditions. To ensure that the real and simulated surfaces felt thermally equivalent, a smooth PVC plate was glued to the tray of the stimulator.

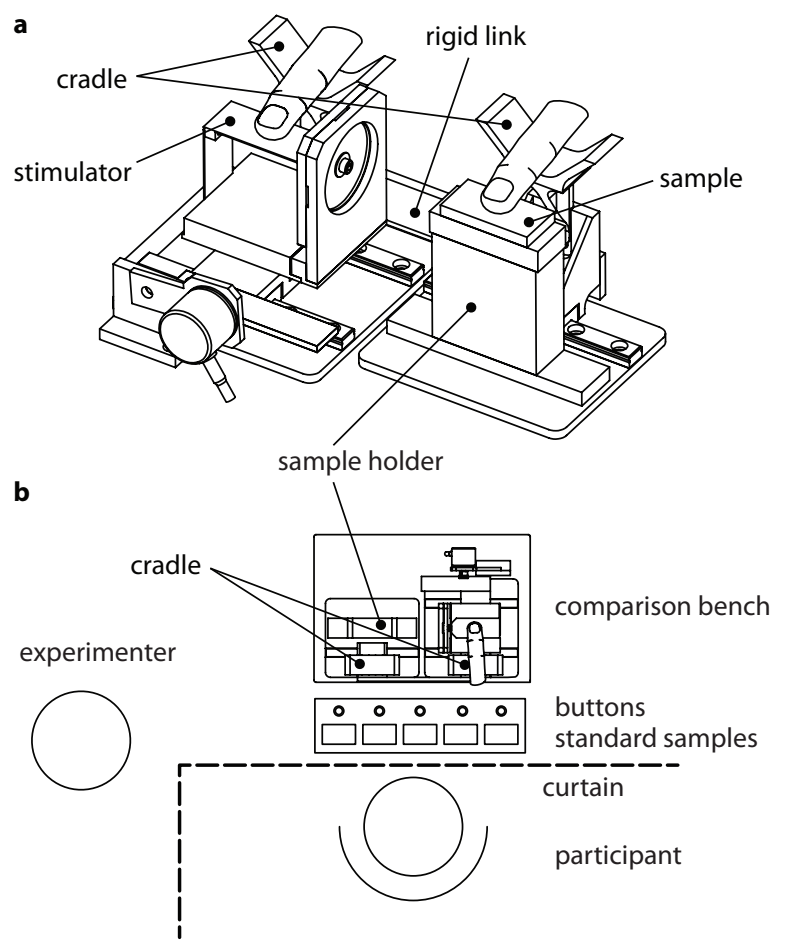

Figure 11. Experimental setup. a. The comparison stimuli bench. The stimulator and a real sample are side by side. During exploration, the participants experienced the stimuli under identical conditions. b. Participants were asked to feel the comparison texture and to match it with the standard textures. They answered by pressing one of the five buttons.

5) Stimuli: Five textured samples were used as stimuli. They were $40 \mathrm{~mm}$ long, $20 \mathrm{~mm}$ wide, $3 \mathrm{~mm}$ thick, and made out of PVC plastic (see Fig. 12). The texture on each sample was created using different machining processes as described in Table II. The resulting micro-geometries were not perceivable without relative motion. The interaction force resulting from scanning with a finger was acquired as described earlier. To do so, one of the participants scanned the surfaces at a constant speed of $50 \mathrm{~mm} / \mathrm{s} \pm 15 \mathrm{~mm} / \mathrm{s}$ and a constant normal force of $0.5 \mathrm{~N} \pm 0.25 \mathrm{~N}$ to match the typical values of natural exploratory movements [37]. Before each measurement a solvent was used to clean the surfaces the participant washed her hand. Recordings were resampled using the method described earlier.
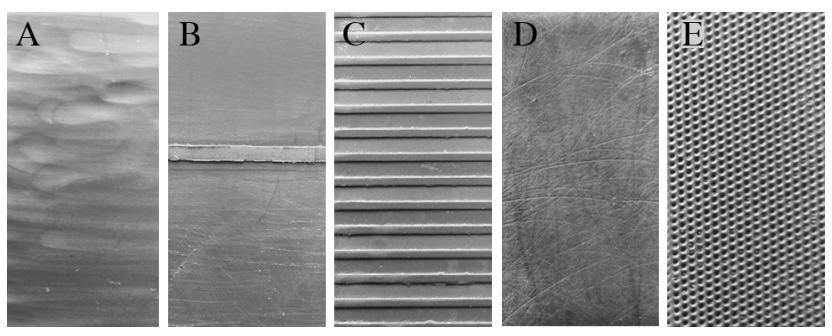

Figure 12. Photos of the five samples.
Table II

MACHINING PROCESSES.

\begin{tabular}{c|lll} 
texture & process & parameters & observations \\
\hline $\mathrm{A}$ & handheld drill & speed: $6000 \mathrm{tr} / \mathrm{min}$ & melted the surface \\
& polishing disk & & \\
\hline $\mathrm{B}$ & milling & speed: $100 \mathrm{tr} / \mathrm{min}$ & $1.5 \mathrm{~mm}$ ridge \\
& $40 \mathrm{~mm}$ endmill & feed rate: $1 \mathrm{~mm} / \mathrm{s}$ & $0.1 \mathrm{~mm}$ high \\
& multiple teeth & depth: $0.1 \mathrm{~mm}$ & \\
\hline $\mathrm{C}$ & milling & speed: $5000 \mathrm{tr} / \mathrm{min}$ & $3 \mathrm{~mm}$ period \\
& $2 \mathrm{~mm}$ endmill & feed rate: $2 \mathrm{~mm} / \mathrm{s}$ & $0.1 \mathrm{~mm}$ deep \\
& two-flute & & grating \\
\hline $\mathrm{D}$ & coarse sandpaper & P40 grade & random scratches \\
\hline $\mathrm{E}$ & drilling with $60^{\circ}$ & depth: $0.1 \mathrm{~mm}$ & quincunx pattern \\
& conical end-mill & $0.5 \mathrm{~mm}$ spacing &
\end{tabular}

6) Participants: Ten volunteers were recruited for the study. They were seven male and three female from the staff of CEA-LIST, aged 25 to 31 . Three of them had experience with haptic devices, but all of them were naive as to the purpose of the experiment. They all were right-handed. They all gave their informed consent and did not report any motor or tactile deficit.

7) Procedure: Participants were placed in front of the setup hidden by a curtain, as illustrated in Fig. 11a. They were explained verbally and by a schematic the overall layout, without disclosing the details of the apparatus and then were explained their task. They then donned acoustic isolation headphones (1015210, Sperian Protection, Roissy-CDG, France) that provided $30 \mathrm{~dB}$ of sound attenuation. White noise was also played through the headphones, and the volume could be adjusted to a comfortable level. There was a small light attached to the curtain to cue the participants to the next trial.

The five real textures samples were placed on a jig right behind the curtain and each was associated to a push-button, see Fig. 11b. Behind the answer panel was the comparison stimulus bench slightly elevated so that the jig did not perturb exploration. The experimenter guided the third phalanx of the participants to rest in the cradles. Three of the five textures, $\mathrm{C}, \mathrm{D}$, and $\mathrm{E}$ were used as comparison stimuli whether real or simulated.

Before each session, all five comparison stimuli, either real or simulated, were presented successively to the participant until they would be familiar with them. They typically became familiar with the stimuli after two rounds, but did not have to memorized them. A group of five participants were presented simulated textures first, and the other five real textures as comparison stimuli first. They were then all tested in the other condition in the second session. During the trials, the comparison stimuli $\mathrm{C}, \mathrm{D}$, and $\mathrm{E}$ were presently randomly. Participants identified the samples by matching them with one of the five standard textures.

When real textures were used as comparison, the experimenter manually changed the sample according to the instructions of the computer. The trials stopped whenever the session duration exceeded 15 minutes, typically after 30 or 40 trials, or when the trial number reached a hundred, whichever came first. In the case of virtual textures, since the process was faster, all participant performed 100 trials under 15 minutes. The volunteers were interviewed after each session to record their subjective experience. 


\section{B. Results}

a

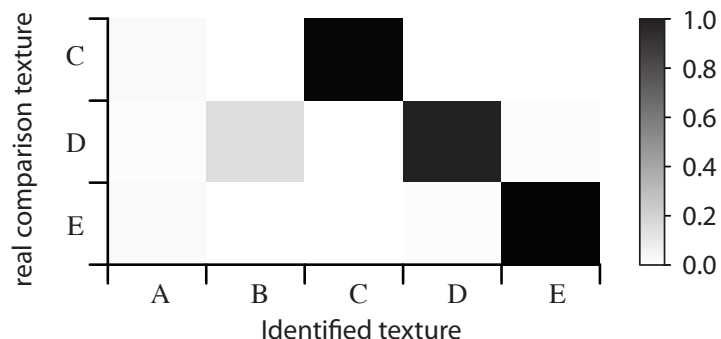

b

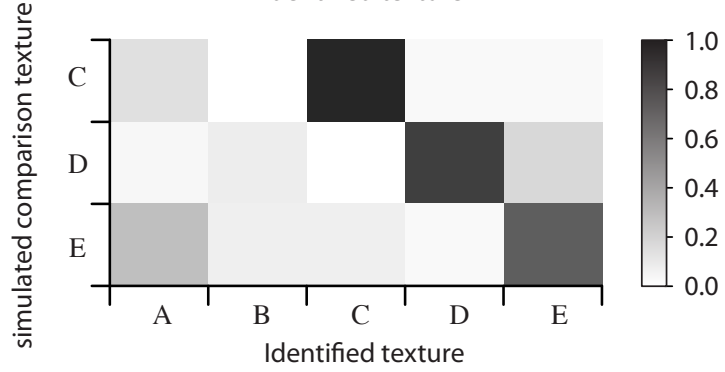

Figure 13. Confusion matrices. a. Matching real textures. b. Matching simulated with real textures. The gray scale represent the proportion of correct answers.

The overall results can be summarized by the confusion matrices shown in Fig. 13. The answer rates are shown by a gray scale from no match (white) to perfect match (black). When the real textures were used as comparison stimuli, Fig. 13a, identification was nearly perfect, which showed that it was possible to identify the samples. There was some confusion with the textures that were not used as comparison. After the experiment, the participants reported that they felt the need to detect them even though they were never presented. All noticed, however, that some samples were missing. When the comparison textures were simulated, Fig. 13b, the pattern was similar and the identification rate high but there was noticeably more confusion between samples A and E.

The rates of success of each participant in the two conditions are presented in Fig. 14. Although no detailed statistics were computed due to the small amount of data, it is apparent that the participants who were tested with the real comparisons first, participants 4,5 , and 6 , performed better than their counterparts, but more importantly, that there was transfer due to learning from the real condition to the simulated condition.

The overall success rate in the real texture condition was 0.93 , with 0.10 of standard deviation. In the simulated texture condition the mean success rate was 0.75 with 0.14 of standard deviation among all participants. The group who performed the task in the real condition first had a success rate of 0.79 with standard deviation of 0.11 in the simulated condition.

Upon debriefing, we learned that all the participants, except one, felt a difference between the two sessions. They also described realistic sensations of rough textures, but the lack of an associated sensation of friction disturbed them somewhat. Six of them noticed the absence of relative motion between the stimulator and their finger. The confusion between sample A and $\mathrm{E}$ seems to be due to the fact that the perceived magnitudes of roughness were almost identical. real texture condition $\square$ simulated texture condition

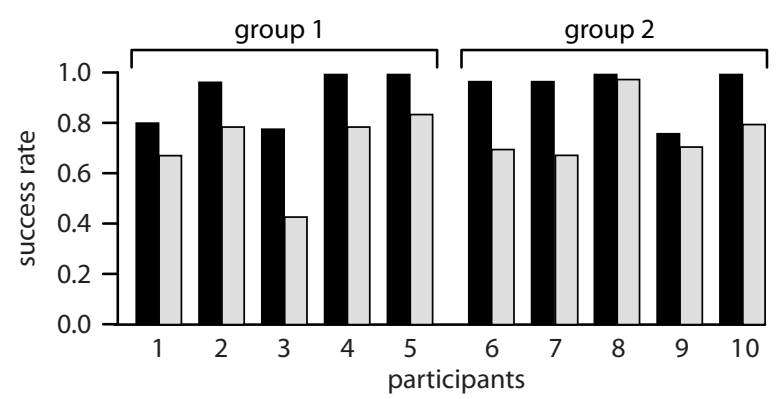

Figure 14. Success rates at the matching experiment with real and digital tactile textures.

\section{Discussion}

The results suggest that the texture recognition task can be adequately performed but say nothing regarding the fidelity of the representation. Key indications regarding the percepual accuracy of the sensations given by the apparatus concern the spectral properties of the stimuli in addition and their intensity. The ability of the apparatus to convey stimulation magnitude was already tested in [31], so we designed an experiment aimed at testing the participants' ability to discriminate the spatial frequency of pure tones.

\section{EXPERIMENT 2: TONE DISCRIMINATION}

We characterized the realism of the display by asking participants to discriminate pure spatial tones and by comparing the results with data found in the literature. The participants were asked to discriminate the frequency of a single sinusoidal grating that of a simulated counterpart. The Weber fraction, extracted from the data, was then compared to the results of [38].

\section{A. Materials and Methods}

1) Participants: Eight volunteers, 6 male and 2 female were recruited from the staff of CEA LIST (age 23 to 31). Two of them had experience with haptic interfaces, but were naive about the purpose of the study. They all were right-handed and did not report any somatosensory deficits. They verbally gave their inform consent.

2) Stimuli: The reference stimulus was a single sinusoidal wave epoxy grating accurately reproduced by a molding process of the very same $1.76 \mathrm{~mm}$-spatial-period and $12.8 \mu \mathrm{m}$ amplitude grating used in [38]. The process used RTV silicon that can reproduce details as small as $1 \mu \mathrm{m}$. The first author's finger response was recorded as described in Experiment 1. The average finger speed was $64 \mathrm{~mm} / \mathrm{s}$ and the normal force was $0.74 \mathrm{~N}$. Comparison stimuli were delivered as previously described, with the difference that the spatial scale was stretched with a ratio $r$. The samples was then truncated and stiched to adjust the length of the records. The amplitudes of the virtual gratings were scaled by the factor $r$ so the slope of the undulation would have the same value as described in [39]. Six stretching factor were tested : $-70 \%,-50 \%,-30 \%,-10 \%$, $+10 \%,+30 \%,+50 \%$ and $+70 \%$, that is, the spatial period of the simulated textured varied from $0.53 \mathrm{~mm}$ to $2.99 \mathrm{~mm}$. 
3) Procedure: A 2-AFC constant stimuli procedure was carried out using the same bench as in Experiment 1, Fig. 11b. The standard stimulus was rigidly bonded to the support of the left of the bench. The comparison stimuli were presented as described earlier. Participants sat behind a curtain that concealed the apparatus and listened to the same white noise. They were ask to sense the texture by resting their finger in a cradle to experience the comparison and the standard stimulus alike. After a short instruction of the task, they could experience the standard stimulus and the comparison stimuli from the smallest spatial period to the largest twice. They were then presented randomly stretched samples and asked to tell which one of the two had the smallest spatial period. They gave their answer by keystrokes. The procedure stopped when all the samples were presented at least 10 times. The total procedure took 30 minutes at most.

4) Analysis: The data analysis described in [38] was carried out. The results were fitted cumulative gaussian distribution using a Maximum Likehood techniques. The 0\% stretching corresponds to the point of subjective equivalence, so its value should correspond to the $50 \%$ proportion. We used a prior on the position of the inflection point of the statistic distribution at $0 \%$ of stretching coefficient. Therefore, only the slope of the distribution was adjusted. The Weber fractions were retrieved from the stretching factor that led to the $75 \%$ threshold. One of the participant, had abnormal results that are not reported.

\section{B. Results and Discussion}

Figure 15 shows the results for each participant. The average Weber fraction is $25.3 \%$ with a standard deviation of $5.8 \%$. Using similar procedure but with real samples, Nefs et al. found a Weber fraction of 15.5\% [38].

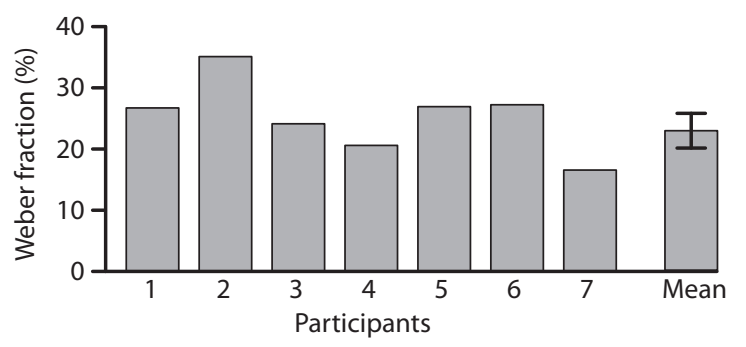

Figure 15. Spatial frequency discrimination performance for all participants.

While the discrimination performance using our apparatus caused a small deficit in performance compared to real samples, the results show that particants could discriminate spatial frequences adequately.

\section{DISCUSSION: IMPLICATIONS FOR HUMAN AND ROBOTIC TOUCH}

This discussion follows directly from the above findings that were obtained from the performance of human participants. In fact, they apply also to robotic touch if robots of the future are to be endowed, like humans, with the faculty to detect, discriminate, and identify textured surfaces instantly. The discussion is organized as a set of observations.

\section{A. The Absence Of Spatially Distributed Information Does Not Imply Temporal Representation}

The experimental conditions in which we placed the participants forced them to base their judgement on just two perceptual cues: stimulation magnitude and spectral content, since spatially distributed information was completely eliminated, as well as low frequency signal components and vertical movements. Yet they performed very well at matching textures and the majority reported a high degree of realism despite missing cues such as relative slip. This observation begs the question of the choice of domain in which spectral content of tactual textures should be represented, implicitly implying the domain in which textures might or should be processed.

One possibility is to represent the mechanical signal of interaction, the rapid skin lateral displacements specifically, in the time domain, like in acoustics. Several facts argue against this option. First, in contrast to acoustics, the representation would depend crucially on the condition under which the signal is acquired, namely on the scanning velocity, and hence would not be invariant, something which is perceptually troublesome. Secondly, while touch is capable of fine temporal discrimination $(5-10 \mathrm{~ms})$, unlike audition, its time-domain spectral processing abilities are poor [40], [41], [42]. In fact, when experiencing the signals that we have collected directly and without correlated movement is not felt by naive participants as texture but as what it is: vibrations. In fact, it is quite difficult to discriminate the textures tactually on the basis of temporal information only.

\section{B. Candidate Representation: The Spatial Spectrograms and The Spatial Formant Organization}

Another option is to represent texture in the space domain, even if it was acquired through regular time intervals, by expressing the interaction force as a function of finger position, that is, using a transformation such as that discussed in Section IV-A3. An analysis in terms of the variation of spectral components through space gives rise to "spatial spectrograms" that express at each point in space the distribution of signal energy in terms of spatial frequencies: from 'smooth to sharp', rather than 'low to high' in the time domain.

The spatial spectrograms of the five standard texture where computed using a short-term fast Fourier transform with a $10 \mathrm{~mm}$ Blackman window and with zero-padding to match the length of the temporal representation. The results can be seen in Fig. 16 where each texture corresponds to a distinctive, highly structured pattern. This fact is quite intriguing. Recall from Fig. 1 that a texture profile, a triangular wave in this case, is converted through scanning into a complex interaction signal by a highly nonlinear transformation, despite the fact that the finger is a soft, deformable, low curvature object, but with high frequency details [12], [43]. After nonlinear transformation by the finger, the five textures are compactly represented by highly distinguishable structured patterns.

Each has a particular "formant" organization, to adopt a notion from acoustics, which are two-dimensional regions of high signal energy and that are not necessarily harmonic or even 
quasi-harmonic. The presence of this organization further argues against the notion of time-domain texture processing. For instance, in texture $\mathrm{B}$, the original $1.5 \mathrm{~mm}$-wide geometrical ridge is tactually represented by a round energy peak, $10 \mathrm{~mm}$ wide and one-decade high. That texture samples A and $\mathrm{E}$ were hardly confused in the real condition but that in the simulated condition texture $\mathrm{E}$ was relatively frequently mistaken for $\mathrm{A}$ is interesting. The corresponding spatial spectrograms do share some common features but are shifted in spatial frequency. It could be that the very low frequencies of A contained spatially distributed information that was eliminated by the experimental conditions. Surprisingly, samples D and E were not confused frequently. Although their spatial formants differ, $\mathrm{E}$ is noticeably stronger than $\mathrm{D}$, thus supporting a two-cue hypothesis. If one cue fails to provide reliable information, the other takes over.

\section{Other Possibilities Based On Neurophysiology}

Of course, other representations would be possible and with appropriate transformations could even be equivalent to the spatial spectrograms. Chiefly among them in the context of textures, are scalograms. These representations would also be compact and informative but have the inconvenience of depending on the arbitrary choice of a particular wavelet function-unless some optimality principle could be invoked. The short-time Fourier transform has the advantage that the only arbitrary parameter is the window length, which is worth discussing. The $10 \mathrm{~mm}$ window used in the short-term Fourier transform was selected simply because it is about the size of the contact surface of a scanning finger. In essence, this means that an isolated spatial feature should give signal inside a $10 \mathrm{~mm}$ window during scanning and be silent outside. Other window sizes could also be based on other optimality properties, for instance, be based the size of receptive fields of particular classes of mechanoreceptors in the skin [44], [45].

\section{CONCLUSION}

We designed an apparatus that was able to record with high precision and wide bandwidth the force of interaction between a finger and a textured surface. We first found by examining the results of scanning a "simple texture" that the interaction mechanics were complex and nonlinear that transform the underlying geometry into a broadband signal with little harmonic connection with the original geometry. We then used this apparatus to record different textures and inverted the process to reproduce as precisely as we could the original vibrations of the skin, but discarding distributed information and normal movements. Participants were still able to identify those textures with a high rate a success and several reported a keen yet imperfect experience of realism. Interestingly, the deficit of realism was not due, consciously, to the absence of distributed information or vertical movement but rather to the absence of the sensation of sliding friction, something we intend to correct in the near future.

For about a century, the notion that tactual texture perception is dependent on the relative sliding of a finger against a surface has been the subject of much discussion. Underlying
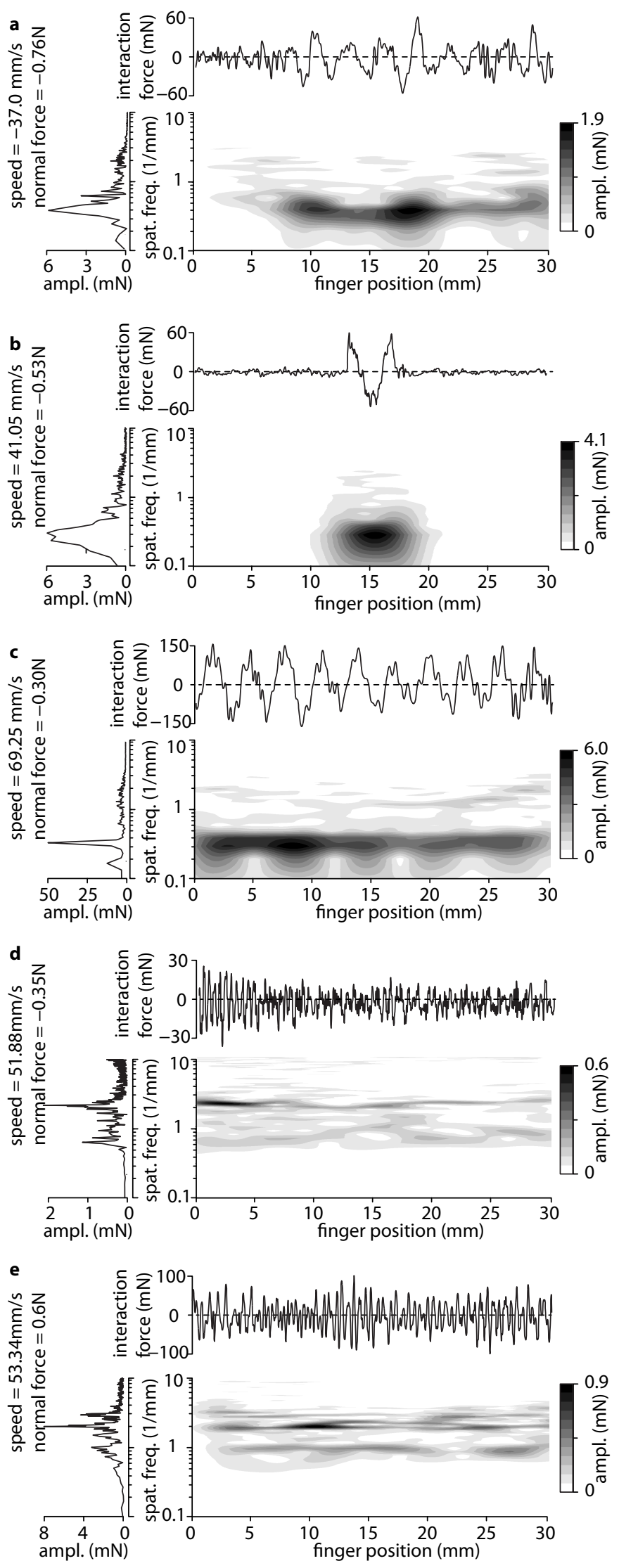

Figure 16. Force-position profile along with the spatial spectrograms (expressed in spatial frequencies and position) and the average spectrum of each measurement made on the original textures. 
this discussion is the assumption that the signals of interest to the sensing organism is a vibration pattern dependent on time, like in acoustics, combined with a spatial detection mechanism distributed on the skin. In our method we stimulated the finger with vibrations arising from a bare finger scanning naturalistic, textured surfaces, but dependent on space, that is, the stimulation depended on how the subject moved which they were free to choose, and there was no information regarding the distribution of stimulation on the skin. We then computed spatial spectrograms using a short-term Fourier transform with a $10 \mathrm{~mm}$ window and found that fingers transformed the original textures into a signal that could be represented as a spatial formant organization that compactly encoded the original surface. Our experiments are not incompatible with the hypothesis that these, or similar space-based transformations, could be employed by the nervous system to identify textures at perceptual speed. Such an approach might also be applicable to texture-aware robots.

\section{ACKNOWLEDGMENT}

The authors would like to thank Edwige Pissaloux for insightful comments and Astrid Kappers for the loan of exquisitely made sinusoidal gratings. This work was supported by the Agence Nationale de la Recherche (ANR) through the REACTIVE project (ANR-07-TECSAN-020).

\section{REFERENCES}

[1] L. E. Krueger, “Tactual perception in historical perspective: David Katz's world of touch," in Tactual Perception; A Sourcebook, W. Schiff and E. Foulke, Eds. Cambridge University Press, 1982, pp. 1-55.

[2] T. R. Thomas, "Characterization of surface roughness," Precision Engineering, vol. 3, no. 2, pp. 97-104, 1981

[3] J. M. Bennett, "Recent developments in surface roughness characterization," Measurement Science and Technology, vol. 3, no. 12, pp. 11191127, 1992.

[4] J. C. Stevens and J. R. Harris, "The scaling of subjective roughness and smoothness," Journal of Experimental Psychology, vol. 64, pp. 498-494, 1962.

[5] M. A. Heller, "Visual and tactual texture perception : Intersensory cooperation," Perception and Psychophysics, vol. 31, pp. 339-344, 1982.

[6] S. J. Lederman, "Tactual roughness perception: Spatial and temporal determinants," Canadian Journal of Psychology, vol. 37, no. 4, pp. 498 511,1983

[7] J. W. Morley and A. W. Goodwin, "Sinusoidal movement of a grating across the monkey's fingerpad: temporal patterns of afferent fiber responses." Journal of Neuroscience, vol. 7, pp. 2181-2191, 1987.

[8] J. S. J. Bolanowski, G. A. Gescheider, R. T. Verrillo, and C. M. Checkosky, "Four channels mediate the mechanical aspects of touch," Journal of Acoustical Society of America, vol. 84, no. 5, pp. 1680-1684, 1988.

[9] C. E. Connor and K. O. Johnson, "Neural coding of tactile texture: Comparison of spatial and temporal mechanisms for roughness perception," Journal of Neuroscience, vol. 12, no. 9, p. 3414, 1992.

[10] M. Hollins, R. Faldowski, S. Rao, and F. Young, "Perceptual dimensions of tactile surface texture: A multidimensional scaling analysis," Perception and Psychophysics, vol. 54, no. 6, pp. 697-705, 1993.

[11] C. J. Cascio and K. Sathian, "Temporal cues contribute to tactile perception of roughness," Journal of Neuroscience, vol. 21, no. 14, pp. 5289-5296, 2001.

[12] A. M. Smith, C. E. Chapman, M. Deslandes, J. S. Langlais, and M. P. Thibodeau, "Role of friction and tangential force variation in the subjective scaling of tactile roughness," Experimental Brain Research, vol. 144, no. 2, pp. 211-223, 2002.

[13] D. Picard, C. Dacremont, D. Valentin, and A. Giboreau, "Perceptual dimensions of tactile textures," Acta psychologica, vol. 114, no. 2, pp. 165-184, 2003.
[14] X. Libouton, O. Barbier, L. Plaghki, and J.-L. Thonnard, "Tactile roughness discrimination threshold is unrelated to tactile spatial acuity," Behavioural Brain Research, vol. 208, pp. 473-478, 2010.

[15] W. M. Bergmann-Tiest and A. M. L. Kappers, "Analysis of haptic perception of materials by multidimensional scaling and physical measurements of roughness and compressibility," Acta Psychologica, vol. 121 , no. 1, pp. 1-20, 2006.

[16] D. T. V. Pawluk and R. D. Howe, "Dynamic lumped element response of the human fingerpad," ASME Journal of BiomechanicalEngineering, vol. 121, pp. 178-184, 1999.

[17] T. C. Pataky, M. L. Latash, and V. M. Zatsiorsky, "Viscoelastic response of the finger pad to incremental tangential displacements," Journal of Biomechanics, vol. 38, pp. 1441-1449, 2005.

[18] Q. Wang and V. Hayward, "In vivo biomechanics of the fingerpad skin under local tangential traction," Journal of Biomechanics, vol. 40, no. 4, pp. 851-860, 2007.

[19] P. H. Warman and A. R. Ennos, "Fingerprints are unlikely to increase the friction of primate fingerpads," The Journal of Experimental Biolology, vol. 212, pp. 2016-2022, 2009.

[20] T. Andre, P. Lefevre, and J. L. Thonnard, "Fingertip moisture is optimally modulated during object manipulation," Journal of Neurophysiology, vol. 103, no. 1, pp. 402-408, 2010.

[21] B. N. J. Persson, "Theory of rubber friction and contact mechanics," Journal of Chemical Physics, vol. 15, no. 8, pp. 3840-3861, 2001.

[22] D. Pai, K. Doel, D. James, J. Lang, J. Lloyd, J. Richmond, and S. Yau, "Scanning physical interaction behavior of 3d objects," in Proceedings of the 28th annual conference on Computer graphics and interactive techniques, 2001, pp. 87-96.

[23] H.-Y. Yao, V. Hayward, and R. E. Ellis, "A tactile magnification instrument for minimally invasive surgery," in Proceedings of MICCAI 2004, ser. Lecture Notes in Computer Science, C. Barillot, D. R. Haynor, and P. Hellier, Eds., vol. 3217, 2004, pp. pp. 89-96.

[24] H. Vasudevan and M. Manivannan, "Recordable haptic textures," in Proceedings of the IEEE International Workshop on Haptic Audio Visual Environments and their Application. HAVE 2006, 2006, pp. 130-133.

[25] J. Lang and S. Andrews, "Measurement-based modeling of contact forces and textures for haptic rendering," IEEE Transactions On Visualization And Computer Graphics, vol. 16, p. in press, 2010.

[26] A. Yamamoto, S. Nagasawa, H. Yamamoto, and T. Higuchi, "Electrostatic tactile display with thin film slider and its application to tactile telepresentation systems," IEEE Transactions on Visualization and Computer Graphics, vol. 12, no. 2, pp. 168-177, 2006.

[27] T. Maeno, K. Otokawa, and M. Konyo, "Tactile display of surface texture by use of amplitude modulation of ultrasonic vibration," in Proceedings of the IEEE Ultrasonics Symposium, 2006, pp. 62-65.

[28] M. Takasaki, H. Kotani, T. Nara, and T. Mizuno, "Transparent surface acoustic wave tactile display," in Proceedings of the IEEE/RSJ International Conference on Intelligent Robots and Systems, 2005, pp. 11151120.

[29] M. Biet, F. Giraud, and B. Lemaire-Semail, "Squeeze film effect for the design of an ultrasonic tactile plate," IEEE Transactions on Ultrasonics, Ferroelectrics and Frequency Control, vol. 54, no. 12, pp. 2678-2688, 2007.

[30] L. Winfield, J. Glassmire, J. E. Colgate, and M. Peshkin, "T-PaD: Tactile pattern display through variable friction reduction," in Proceedings of the Second Joint EuroHaptics Conference and Symposium on Haptic Interfaces for Virtual Environment and Teleoperator Systems, World Haptics 2007, 2007, pp. 421-426.

[31] M. Wiertlewski, J. Lozada, E. Pissaloux, and V. Hayward, "Causality inversion in the reproduction of roughness," in Proceedings of Europhaptics 2010, ser. LNCS, A. M. L. Kappers et al., Ed., vol. 6192, no. II. Springer-Verlag, 2010, pp. 17-24.

[32] M. Wiertlewski, C. Hudin, and V. Hayward, "On the 1/f noise and noninteger harmonic decay of the interaction of a finger sliding on flat and sinusoidal surfaces," in Proceedings of the Fourth Joint Eurohaptics Conference and Symposium on Haptic Interfaces for Virtual Environment and Teleoperator Systems, 2011, in press.

[33] G. Millet, S. Haliyo, S. Regnier, and V. Hayward, "The ultimate haptic device: First step," in Proceedings of the Third Joint Eurohaptics Conference and Symposium on Haptic Interfaces for Virtual Environment and Teleoperator Systems, 2009, pp. 273-278.

[34] J. G. Smits, S. I. Dalke, and T. K. Cooney, "The constituent equations of piezoelectric bimorphs," Sensors and Actuators A: Physical, vol. 28, pp. 41-61, 1991.

[35] M. Cruz-Hernandez and V. Hayward, "Phase control approach to hysteresis reduction," IEEE Transactions On Control Systems Technology, vol. 9, no. 1, pp. 17-26, 2001 . 
[36] G. Campion and V. Hayward, "Fundamental limits in the rendering of virtual haptic textures," in Proceedings of the First Joint Eurohaptics Conference and Symposium on Haptic Interfaces for Virtual Environment and Teleoperator Systems, 2005, pp. 263-270.

[37] A. M. Smith, G. Gosselin, and B. Houde, "Deployment of fingertip forces in tactile exploration," Experimental Brain Research, vol. 147, pp. 209-218, 2002.

[38] H. T. Nefs, A. M. L. Kappers, and J. J. Koenderink, "Amplitude and spatial-period discrimination in sinusoidal gratings by dynamic touch," Perception, vol. 30, pp. 1263-1274, 2001.

[39] S. Louw, A. Kappers, and J. Koenderink, "Haptic detection thresholds of Gaussian profiles over the whole range of spatial scales," Experimental brain research, vol. 132, no. 3, pp. 369-374, 2000.

[40] G. D. Goff, "Differential discrimination of frequency of cutaneous mechanical vibration," Journal of Experimental Psychology, vol. 74, no. 2, pp. 294-299, 1967.

[41] S. J. Bensmaïa and M. Hollins, "Complex tactile waveform discrimination," Journal of Accoustical Society of America, vol. 108, no. 3, pp. $1236-1245,2000$.

[42] D. A. Mahns, N. M. Perkins, V. Sahai, L. Robinson, and M. J. Rowe, "Vibrotactile frequency discrimination in human hairy skin," Journal of Neurophysiology, vol. 95, no. 3, pp. 1442-1450, 2005.

[43] J. Scheibert, S. Leurent, A. Prevost, and G. D. G., "The role of fingerprints in the coding of tactile information probed with a biomimetic sensor," Science, vol. 323, pp. 1503-1506, 2009.

[44] Å. V. Vallbo and R. S. Johansson, "Properties of cutaneous mechanoreceptors in the human hand related to touch sensation," Human Neurobiology, vol. 3, pp. 3-14, 1984.

[45] R. H. LaMotte and J. Whitehouse, "Tactile detection of a dot on a smooth surface: peripheral neural events," Journal of Neurophysiology, vol. 56, pp. 1109-1128, 1986.

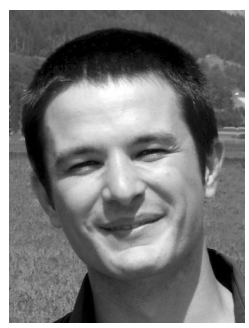

Michael Wiertlewski earned a Master's degree in Image, Vision, and Signal Processing from the Université Jean Monnet, Saint Etienne, France and a Diplôme d'ingénieur from Ecole Nationale d'Ingénieurs de Saint-Etienne (ENISE) both in 2007. $\mathrm{He}$ is currently pursuing a Ph.D. degree at the Université Pierre et Marie Curie under the auspices of the Laboratoire d'Intégration des Systèmes et des Technologies of the Commissariat à l'Energie Atomique (CEA-LIST) in Fontenay-aux-Roses, France. His research interest include the design of tactile interfaces, virtual reality environments, and the perception of tactual textures. $\mathrm{He}$ is the recipient of the Eurohaptics 2010 Best Paper Award.

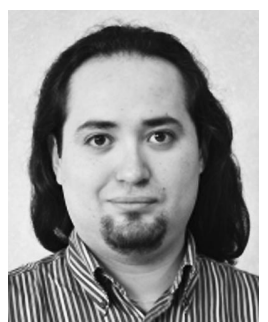

José Losada was awarded a Master of Science in Robotics from Ecole Normale Supérieure, Paris, France, and a Ph.D. from Ecole Polytechnique, Paris, France, in 2003 and 2007 respectively. He then joined the Sensory and Ambient Interfaces Group within the Laboratoire d'Intégration des Systèmes et des Technologies of the Commissariat à l'Energie Atomique (CEA-LIST) in Fontenay-auxRoses, France, as project leader. His current research interests are in the area human-machine interfaces and mechatronic systems with an emphasis on haptic interfaces, tactile interfaces, and devices based on smart materials. He holds 7 patents and published 10 articles on these subjects.

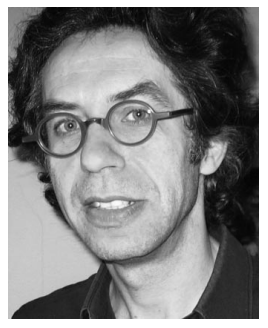

Vincent Hayward (M'84-SM'04-FIEEE'08, DrIng., 1981 Univ. de Paris XI) was Postoctoral Fellow (1982-83) at Purdue University, and joined CNRS, France, as Chargé de Recherches in 1983. In 1987, he joined the Department of Electrical and Computer Engineering at McGill University as assistant, associate and then full professor (2006) and was the Director of the McGill Center for Intelligent Machines from 2001 to 2004. Hayward co-founded spin-off companies and received several best paper and research awards. He is on editorial board of the ACM Transaction on Applied Perception and of the IEEE Transactions on Haptics and is a Fellow of the IEEE. As of 2008, he holds the "chaire internationale d'haptique' at the Université Pierre et Marie Curie. 